\title{
Security and Risk Analysis of Financial Industry Based on the Internet of Things
}

\author{
Yizhi Li (iD) ${ }^{1,2}$ \\ ${ }^{1}$ Weatherhead School of Management, Case Western Reserve University, Cleveland 44106, OH, USA \\ ${ }^{2}$ Dornslife School of Arts and Science, University of Southern California, Los Angeles 90089, CA, USA \\ Correspondence should be addressed to Yizhi Li; lijasmin@usc.edu
}

Received 18 November 2021; Revised 29 December 2021; Accepted 20 January 2022; Published 21 February 2022

Academic Editor: Deepak Kumar Jain

Copyright (c) 2022 Yizhi Li. This is an open access article distributed under the Creative Commons Attribution License, which permits unrestricted use, distribution, and reproduction in any medium, provided the original work is properly cited.

Internet of Things finance is a new type of financial industry in which Internet of Things enterprises and financial institutions borrow information and communication technology and Internet of Things technology and use algorithms and models to realize payment, positioning, investment, measurement, capital financing, asset management, and information intermediary services. The purpose of this paper is to implement risk management and control based on the Internet of Things by enumerating, comprehending, and all-embracing data, effectively improve reliability and efficiency, and minimize risks under certain conditions. First this article on the Internet of things and Internet of things played a role in financial giving relevant summary and interpretation of the analysis of the present condition, and the rate of financial security and risk is based on Internet environment, and for financial security and risk measurement, positioning and computing scheme, attached to the unit and multiple positioning and Taylor calculation model, is used to determine and control the financial risk. In addition, beBS-T encryption model is used to encrypt financial nodes to strengthen financial security and reduce financial risks. AHP is then used to establish a risk judgment matrix to evaluate the financial system and calculate the index of risk weight. After that, simulation algorithm is used to check nodes and risk measurement experiments. The improved ant colony algorithm NACO is compared with the basic algorithm TACO, and the further improved TSN metric algorithm with increment is proposed. The conclusion is drawn as follows: The improved ant colony algorithm NACO is $23.46 \%$ lower than the basic ant colony algorithm TACO in the failure jitter end of risk measurement, while the incremental I-NACO algorithm shows better utility and is $7.1 \%$ lower than the NACO algorithm in the delay jitter of risk measurement. The Internet of Things is taking a deep role in the financial field, and its shape is in the making. In the process of controlling financial risks, it can make a great contribution to gradually innovate financial forms and reform business models.

\section{Introduction}

1.1. Background. In recent years, the same generation of high-end technology, the target in the bank's security system, and willful, against the wind crime, not only customer information stolen, but also the loss of funds, reduce the credibility of the bank, for this bad behavior, based on the Internet of things environment, and conduct research and analysis on asset management, monitoring, and measuring risks, abnormal behavior blocking, algorithmic encryption financial security, and intrusion detection, in order to optimize the application of the Internet of Things in finance. Internet of Things is a connection between people and
Things that take the Internet and mobile communication as the media. There are a large number of communication devices in the Internet of Things, which can realize the interconnection of things through communication interfaces and guide the intelligent control of various objects through specific logic control. Meanwhile, a series of algorithms and models can realize the measurement of financial risks. Attackers access IoT devices through the Internet, which can easily make the IoT vulnerable to malicious attacks from external sites. In this case, prevention technologies can be added through positioning monitoring, encryption algorithms, node identification, risk measurement, and other methods to improve the security of IoT 
devices. To reduce risk, however, some algorithm can produce high complexity, or measurement is not accurate, consuming a large amount of resources; at this point, the optimization of the encryption algorithm and risk measurement algorithm undoubtedly becomes the effort direction; for example, the Internet of things has very many algorithms and the model and optimization algorithm are verified through the experiment, and the financial risk analysis is done under the Internet of things.

1.2. Significance. The purpose of this paper is that the Internet of the financial risk control testing calculation measures beneficial exploration in the financial risk; there are some measurement methods and models, although with a good calculation data, but some data is not accurate; aging cycle extension is long; this article on the analysis of the algorithm and the application of the model in the aspect of financial risks reconstructs the incremental scheduling algorithm to be more perfect, and based on this design experiment, statistical data verify and strive to achieve higher efficiency and more accurate control of financial risks in the environment based on the Internet of things, in order to have a certain guiding significance in financial security and risk analysis.

1.3. Related Work. The Internet of Things is like a nectar injected into the development of science and technology, causing the prosperity of the society to develop. Based on the seamless interaction between a large number of heterogeneous devices, the Internet of Things uses its advanced nature, security, and reliability and continues to be used in the financial industry. With the in-depth exploration of the Internet of Things, scientists in various fields have spoken one after another. Palattella M R stated that the IoT paradigm is expected to completely change the way we live and work through a large number of new services based on the seamless interaction between a large number of heterogeneous devices. After decades of development, the concept of the Internet of Things has gradually appeared in a wide variety of communication technologies in recent years, reflecting the huge diversity of application fields and communication requirements [1]. Perera $C$ shows that the Internet of Things (IoT) is a dynamic global information network composed of objects connected to the Internet, such as radio frequency identification, sensors, actuators, and other instruments and smart appliances that are becoming an integral part of the future Internet [2]. Hyun-sik uses a scenario-based loss measurement model based on FAIR to identify the extent of damage and calculates the reasonable damage considered to be an ISMS problem. We apply the current ISMS and ISO 27001 control items instead of modifying ISMS to identify the financial impact of ransomware loss and risk of the industry and ways to reduce losses [3]. Fu Y used 31 provinces and cities in China as research objects, established a financial industry competitiveness evaluation index system, and compared the financial industry in the Beijing-Tianjin-Hebei region with the financial industry in the Yangtze River Delta. The results show that the scale, financial performance, financial safety, and radiation capacity of Beijing's financial industry are relatively high, and Hebei's financial industry relies on Beijing-Tianjin radiation [4]. Gai $\mathrm{K}$ proposed a secure cyber incident analysis framework using big data, called Cost Aware Hierarchical Cyber Incident Analysis (CAHCIA). This method is designed to match different cyber risk scenarios using repository data and use Monte Carlo simulation to extract event features based on the training data set [5]. Sato $\mathrm{R}$ showed the design and experimental results of a fast single-flux quantum (RSFQ) bit serial microprocessor, which has a reduced embedded random access memory (RAM) and a minimum instruction set, called CORE e2h [6]. Filipe uses fault injection and heavy ion experiments to study the impact of register file errors on the reliability of modern embedded microprocessors. In addition, we evaluated how different levels of compiler optimization modify the use of processor register files and the probability of failure [7]. Since 2020, external security incidents of the Internet of Things have emerged one after another. According to data provided by the Critical Infrastructure Security Emergency Response Center, as of July, 7.46 million device addresses were attacked in China, an increase of $9.5 \%$ compared to January. However, the current IoT positioning and algorithm models have a long time extension period, and the accuracy needs to be enhanced. This is also the drawback of analyzing financial risks in the IoT environment.

1.4. Innovation. Main purpose of this article is to study, based on Internet environment, interpretation of the analysis of financial safety and risk, under the Internet of various methods of the docking financial effect and function, and for financial security and risk measurement, such as orientation, Taylor BEBS structuring model, $T$ encryption method, AHP analytic hierarchy process (AHP) risk judgment matrix, evaluation of the financial system, calculate the risk weighting of the indicators and simulate the algorithm degree check node and risk measurement experiment, compare the improved ant colony algorithm NACO and the basic algorithm TACO, further put forward the improved algorithm with increment TSN measurement, through method calculation, experimental analysis, detection data, and verify its feasibility, in financial security and risk which can bring targeted optimization.

\section{Financial Security and Risk Analysis Methods Based on the Internet of Things}

\subsection{The Internet of Things Monitoring and Positioning Method}

2.1.1. Unit Variable Taylor IoT Monitoring Risk Positioning. Among the node location algorithms of the Internet of Things, Taylor series location method is a common iterative 
location algorithm that can be used for locating risks. It is characterized by high precision and fast convergence [8]. The solution process of the unfolding model of Taylor's level localization method is solved by using the trilateral ranging method. This is shown in Figure 1.

Assuming that $m+n$ sensor nodes are randomly and uniformly deployed in a two-dimensional space, there are unknown nodes, and there are $b$ anchor nodes. The real position of the unknown risk point is expressed as $\left(a_{x}, b_{z}\right) \cdot z \in(1,2, \ldots, m)$, and the real position of the anchor node is expressed as $\left(x_{i}, y_{i}\right) \cdot i \in(1,2, \ldots, n)$. The TOA ranging technology is used to measure all nodes, and the measured distances obtained include measurement errors. Node positioning is to calculate the estimated coordinates of the unknown node based on the obtained measured distance and the known anchor node coordinates and make it as close as possible to the true position of the unknown node [9].

Suppose $\left(x_{z}, y_{z}\right) \cdot z \in(1,2, \ldots, a)$ is the a-th unknown node to be located, and the distance between it and all anchor nodes.

The measurement distances are

$$
\begin{aligned}
& d_{z, 1}=\sqrt{\left(a_{z}-x_{1}\right)^{2}+\left(b_{z}-y_{1}\right)^{2}}+e_{z, 1}, \\
& d_{z, i}=\sqrt{\left(a_{z}-x_{i}\right)^{2}+\left(b_{z}-y_{i}\right)^{2}}+e_{z, i}, \\
& d_{z, n}=\sqrt{\left(a_{z}-x_{n}\right)^{2}+\left(b_{z}-y_{n}\right)^{2}}+e_{z, n},
\end{aligned}
$$

where $\sqrt{\left(a_{z}-x_{i}\right)^{2}+\left(b_{z}-y_{i}\right)^{2}}$ represents the true distance between the unknown node and the anchor node and the address. $d_{z, i}$ represents the measured distance between the node and the anchor node. $e_{z, i}$ indicates the distance measurement error between the unknown node and the anchor node.

Suppose it obeys a Gaussian distribution with mean zero and variance $\sigma_{z, i}^{2}$. Knowing the initial value of the unknown node $\left(\widehat{a}_{z}, \widehat{b}_{z}\right)$, perform Taylor series expansion on the true distance in the above formula and ignore the components above the second order; there are

$$
\begin{aligned}
& d_{z, 1}=\widehat{d}_{z, 1}+\Delta a_{z} \frac{\widehat{a}_{z}-x_{1}}{\widehat{d}_{z, 1}}+\Delta b_{z} \frac{\widehat{b}_{z}-y_{1}}{\widehat{d}_{z, 1}}+e_{z, 1}, \\
& d_{z, i}=\widehat{d}_{z, i}+\Delta a_{z} \frac{\widehat{a}_{z}-x_{i}}{\widehat{d}_{z, i}}+\Delta b_{z} \frac{\widehat{b}_{z}-y_{i}}{\widehat{d}_{z, i}}+e_{z, i}, \\
& d_{z, n}=\widehat{d}_{z, n}+\Delta a_{z} \frac{\widehat{a}_{z}-x_{n}}{\widehat{d}_{z, n}}+\Delta b_{z} \frac{\widehat{b}_{z}-y_{n}}{\widehat{d}_{z, n}}+e_{z, n} .
\end{aligned}
$$

Among them, $\widehat{d}_{z, i}=\sqrt{\left(\widehat{a}_{z}-x_{i}\right)^{2}+\left(\hat{b}_{z}-y_{i}\right)^{2}}$ represents the estimated distance $\Delta a_{z}=a_{z}-\widehat{a}_{z}, \Delta b_{z}=b_{z}-\widehat{b}_{z}$ between the unknown node and the anchor node. After shifting the terms (2)-(4), the matrix form is obtained:

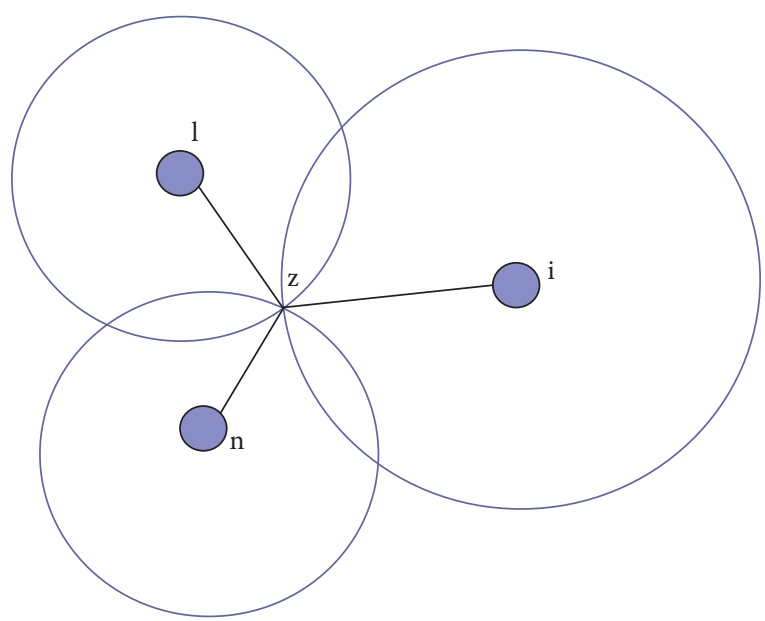

FIgURe 1: Positioning method of trilateral measurement.

$$
\left[\begin{array}{c}
d_{z, 1}-\widehat{d}_{z, 1} \\
\vdots \\
d_{z, i}-\widehat{d}_{z, i} \\
\vdots \\
d_{z, n}-\widehat{d}_{z, n}
\end{array}\right]=\left[\begin{array}{cc}
\frac{\widehat{a}_{z}-x_{1}}{\widehat{d}_{z, 1}} & \frac{\widehat{b}_{z}-y_{1}}{\widehat{d}_{z, 1}} \\
\vdots & \vdots \\
\frac{\widehat{a}_{z}-x_{i}}{\widehat{d}_{z, i}} & \frac{\widehat{b}_{z}-y_{i}}{\widehat{d}_{z, i}} \\
\vdots & \vdots \\
\frac{\hat{a}_{z}-x_{n}}{\widehat{d}_{z, n}} & \frac{\hat{b}_{z}-y_{n}}{\hat{d}_{z, n}}
\end{array}\right]\left[\begin{array}{c}
\Delta a_{z} \\
\Delta b_{z}
\end{array}\right]+\left[\begin{array}{c}
e_{z, 1} \\
\vdots \\
e_{z, i} \\
\vdots \\
e_{z, n}
\end{array}\right] .
$$

Available from (5), $D_{1}=C \Delta_{1}+E_{1}$.

Using the least square method to solve the positioning model, the estimated position of the $\mathrm{z}$-th unknown risk node can be obtained. In financial risks, this Internet of Things calculation method can analyze and calculate a single node. It can effectively capture risks in monitoring positioning. If it is to analyze and locate multiple nodes, it is necessary to use a multivariate algorithm.

\subsubsection{Multivariate Taylor IoT Monitoring Risk Positioning.} The traditional Taylor location algorithm only considers the distance between the unknown risk node and the anchor node and cannot monitor the location of multiple unknown risk nodes in the same condition [10].

In order to make better use of the existing positioning information to further enhance the positioning accuracy of unknown risk nodes and to consider the distance between unknown risk nodes, a new risk positioning model based on the multivariate Taylor positioning algorithm can be established. Equations (6)-(11) are model descriptions: 


$$
\begin{aligned}
d_{1,1} & =\phi_{1,1}\left(a_{1}, b_{1}, \ldots, a_{m}, b_{m}\right)+e_{1,1}, \\
d_{z, i} & =\phi_{z, i}\left(a_{1}, b_{1}, \ldots, a_{m}, b_{m}\right)+e_{z, i}, \\
d_{m, n} & =\phi_{m, n}\left(a_{1}, b_{1}, \ldots, a_{m}, b_{m}\right)+e_{m, n}, \\
d_{1,2}^{\prime} & =f_{1,2}\left(a_{1}, b_{1}, \ldots, a_{m}, b_{m}\right)+e_{1,2}^{\prime}, \\
d_{z, j}^{\prime} & =f_{z, j}\left(a_{1}, b_{1}, \ldots, a_{m}, b_{m}\right)+e_{z, j}^{\prime}, \quad z<j, \\
d_{(m-1), m}^{\prime} & =f_{(m-1), m}\left(a_{1}, b_{1}, \ldots, a_{m}, b_{m}\right)+e_{(m-1), m}^{\prime} .
\end{aligned}
$$
Among
$\phi_{z, i}\left(a_{1}, b_{1}, \ldots, a_{m}, b_{m}\right)=\sqrt{\left(a_{z}-x_{i}\right)^{2}+\left(b_{z}-y_{i}\right)^{2}}$ and $d_{z, i}$ represent the true distance and the measured distance between the anchor node and the unknown node, and $f_{z, j}\left(a_{1}, b_{1}, \ldots, a_{m}, b_{m}\right)=\sqrt{\left(a_{z}-a_{j}\right)^{2}+\left(b_{z}-b_{j}\right)^{2}}$ and $d_{z, j}$ represent the true distance and the measured distance between the unknown node $\left(a_{z}, b_{z}\right)$ and the unknown node $\left(a_{j}, b_{j}\right) . e_{z, i}$ represents the distance measurement error between the anchor node and the unknown node, assuming that it obeys a Gaussian distribution with a mean value of zero and a variance of $\sigma_{z, i}^{2} . e_{z, j}{ }^{\prime}$ represents the distance measurement error between unknown nodes, assuming that it obeys a Gaussian distribution with a mean value of zero and a variance of $\sigma_{\mathrm{z}, \mathrm{j}}^{2}$.

After shifting the terms (7)-(12), we get

$$
\begin{aligned}
& d_{1,1}-\widehat{d}_{1,1}=\Delta a_{1} \frac{\widehat{a}_{1}-x 1}{\widehat{d}_{1,1}}+\Delta b_{1} \frac{\widehat{b}_{1}-y_{1}}{\widehat{d}_{1,1}}+e_{1,1} \\
& d_{z, i}-\widehat{d}_{z, i}=\Delta a_{z} \frac{\widehat{a}_{z}-x_{i}}{\widehat{d}_{z, i}}+\Delta b_{z} \frac{\widehat{b}_{z}-y_{i}}{\widehat{d}_{z, i}}+e_{z, i} \\
& d_{m, n}-\widehat{d}_{m, n}=\Delta a_{m} \frac{\widehat{a}_{m}-x_{n}}{\widehat{d}_{m, n}}+\Delta b_{n} \frac{\widehat{b}_{m}-y_{n}}{\widehat{d}_{m, n}}+e_{m, n} \\
& d_{1,2}^{\prime}-\widehat{d}_{1,2}^{\prime}=\Delta a_{1} \frac{\widehat{a}_{1}-\widehat{a}_{2}}{\widehat{d}_{1,2}^{\prime}}+\Delta b_{1} \frac{\widehat{b}_{1}-\widehat{b}_{2}}{\widehat{d}_{1,2}^{\prime}}-\Delta a_{2} \frac{\widehat{a}_{1}-\widehat{a}_{2}}{\widehat{d}_{1,2}^{\prime}}-\Delta b_{2} \frac{\widehat{b}_{1}-\widehat{b}_{2}}{\widehat{d}_{1,2}^{\prime}}+e_{1,2}^{\prime}, \\
& d_{z, j}^{\prime}-\widehat{d}_{z, j}^{\prime}=\Delta a_{z} \frac{\widehat{a}_{z}-\widehat{a}_{j}}{\widehat{d}_{z, j}^{\prime}}+\Delta b_{z} \frac{\widehat{b}_{z}-\widehat{b}_{j}}{\widehat{d}_{z, j}^{\prime}}-\Delta a_{j} \frac{\widehat{a}_{z}-\widehat{a}_{j}}{\widehat{d}_{z, j}^{\prime}}-\Delta b_{j} \frac{\widehat{b}_{z}-\widehat{b}_{j}}{\widehat{d}_{z, j}^{\prime}}+e_{z, j}^{\prime}, \quad z<j, \\
& d_{(m-1), m}^{\prime}-\widehat{d}_{(m-1), m}^{\prime}=\Delta a_{(m-1)} \frac{\widehat{a}_{(m-1)}-\widehat{a}_{m}}{\widehat{d}_{(m-1), m}^{\prime}}+\Delta b_{(m-1)} \frac{\widehat{b}_{(m-1)}-\widehat{b}_{m}}{\widehat{d}_{(m-1), m}^{\prime}}-\Delta a_{m} \frac{\widehat{a}_{(m-1)}-\widehat{a}_{m}}{\widehat{d}_{(m-1), m}^{\prime}}-\Delta b_{m} \frac{\widehat{b}_{(m-1)}-\widehat{b}_{m}}{\widehat{d}_{(m-1), m}^{\prime}}+e_{(m-1), m}^{\prime} .
\end{aligned}
$$

$$
\left[\begin{array}{c}
d_{1,1}-\widehat{d}_{1,1} \\
\vdots \\
d_{z, i}-\widehat{d}_{z, i} \\
\vdots \\
d_{m, n}-\widehat{d}_{m, n} \\
d_{1,2}^{\prime}-\widehat{d}_{1,2} \\
\vdots \\
d_{z, i}^{\prime}-\widehat{d}_{z, i} \\
\vdots \\
d_{(m-1), m}^{\prime}-\widehat{d}_{(m-1), m}
\end{array}\right]=Q\left[\begin{array}{c}
\Delta a_{1} \\
\Delta b_{1} \\
\vdots \\
\Delta a_{z} \\
\Delta b_{z} \\
\vdots \\
\Delta a_{m} \\
\Delta b_{m}
\end{array}\right]+\left[\begin{array}{c}
e_{1,1} \\
\vdots \\
e_{z, i} \\
\vdots \\
e_{m, n} \\
e_{1,2}^{\prime} \\
\vdots \\
e_{z, j}^{\prime} \\
\vdots \\
e_{(m-1), m}^{\prime}
\end{array}\right] .
$$

This can be written as a positioning model: $D=Q \Delta+E$.

Among them, $\Delta$ is the position deviation matrix $2 m \times 1$, which is a matrix. $E$ is the distance measurement error matrix. $D$ is the distance matrix, which is a matrix $\left(C_{m}^{2}+C_{m}^{1} C_{n}^{1}\right) \times 1$, each element of which represents the difference between the measured value and the estimated value of the distance between all nodes. Each element is used; $d_{z, i}-\widehat{d}_{z, i}$ ( $\mathrm{z}$ is an unknown node, $i$ is an anchor node) or $d_{z, j}^{\prime \prime}-\widehat{d}_{z, j}^{\prime}(\mathrm{z}, j$ are both Unknown node) is represented as a matrix $\left(C_{m}^{2}+C_{m}^{1} C_{n}^{1}\right) \times 2 m$. If the $\mathrm{p}$-th element in $D$ is 
$d_{z, i}-\hat{d}_{z,}$, then the $\mathrm{p}$-th row and the $\mathrm{p}$-th element are $\widehat{a}_{z}-x_{i} / \widehat{d}_{z, i}, \widehat{b}_{z}-y_{i} / \widehat{d}_{z, i}$. The least square method can be used to solve the problem. In order to explain the positioning model more clearly, we can give an example. Suppose there are six nodes, of which there are three unknown nodes. Suppose the unknown nodes are $\left(a_{1}, b_{1}\right),\left(a_{2}, b_{2}\right),\left(a_{3}, b_{3}\right)$, and the anchor nodes are $\left(x_{1}, y_{1}\right),\left(x_{2}, y_{2}\right),\left(x_{3}, y_{3}\right)$. Knowing the initial value of the unknown node $\left(\widehat{a}_{1}, \widehat{b}_{1}, \widehat{a}_{2}, \widehat{b}_{2}, \widehat{a}_{3}, \widehat{b}_{3}\right)$, ignoring the components above the second order, the positioning model can be obtained. $D=Q \Delta+E$.

Among $\Delta=\left[\Delta a_{1}, \Delta b_{1}, \Delta a_{2}, \Delta b_{2}, \Delta a_{3}, \Delta b_{3}\right]^{T}$

$D=\left[d_{1,1}-\hat{d}_{1,1}, d_{1,2}-\hat{d}_{1,2}, d_{1,3}-\hat{d}_{1,3}, d_{2,1}-\hat{d}_{2,1}, d_{2,2}-\hat{d}_{2,2}, d_{2,3}-\hat{d}_{2,3}\right.$

$\left.d_{3,1}-\widehat{d}_{3,1}, d_{3,2}-\hat{d}_{3,2}, d_{3,3}-\hat{d}_{3,3}, d_{1,2}^{\prime}-\hat{d}_{1,2}^{\prime}, d_{1,3}^{\prime}-\hat{d}_{1,3}^{\prime}, d_{2,3}^{\prime}-\hat{d}_{2,3}^{\prime}\right]^{T}$,

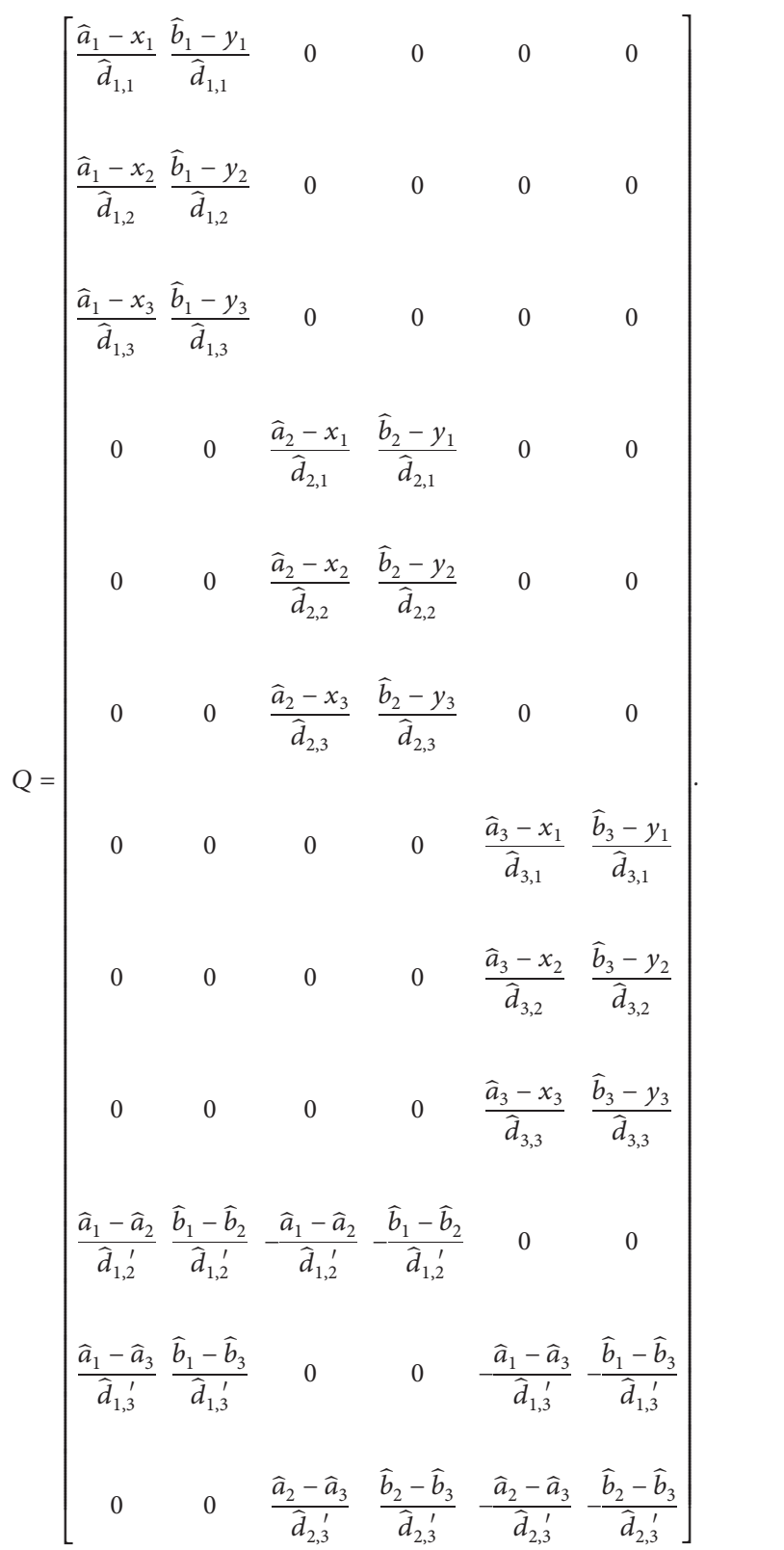

By solving the model, the position estimates of all unknown nodes can be obtained simultaneously. Make full use of the information between nodes, increase the constraints between each other, and improve the accuracy of monitoring positioning, so as to monitor financial risks and reduce risks.

To verify the effectiveness of the Taylor localization algorithm for multiple variables, the time complexity of the algorithm was examined according to the procedure of the algorithm, and the complexity of the traditional trilateral algorithm was compared with that of the new algorithm, and it was concluded that the complexity of the new algorithm for the improved Taylor trilateral measurement was greater than that of the traditional Taylor algorithm.

In node location of Internet of Things, Taylor series expansion method is a commonly used iterative location algorithm, which has the characteristics of high solution accuracy and fast convergence [11]. The principle is shown in Figure 2.

2.2. BEBS-T Encryption Model. The overall structure of the BEBS-T system is composed of the following six parts: (1) APP module; (2) configuration file module; (3) transaction message processing module; (4) communication module; (5) functional interface module; (6) security module. The smooth operation of the BEBS-T system can only be ensured when these six modules work together [12].

The security of financial industry cannot be separated from the support of network technology, password management, and other multidisciplinary, and password technology is the core technology of financial industry security. The PSAM card three-level distributed key is used to encrypt transaction data. In the process of data encryption and decryption in the BEBS-T system, a total of five types of keys are used. They are root key, master key, password encryption key, MAC key, and three-level decentralized key [13]. The specific key relationship is shown in Figure 3.

Encryption process: The encryption processing of transaction data requires the participation of the keyboard and PSAM card. The keyboard mainly encrypts the user password and calculates the MAC of the packet, while the three-level decentralized encryption of PSAM card is between the completion of the transaction packet group and the calculation of the MAC and encrypts the data. Even if the attacker obtains the transaction data, he must also know the PIN key, the MAC key, and the three-level scatter key of the PSAM card to get the clear text of the transaction data [5]. In the past, the self-service terminal only used the encrypted keyboard to complete user password encryption and calculate packet MAC, without the participation of PSAM card. Therefore, the security of the system has been greatly improved in the transaction process.

The schematic diagram of the specific encryption process is as Figure 4.

The three-level decentralized encryption process of the PSAM card is as follows:

(1) First, use the bank branch number as the key, and perform a 3DES encryption on the transaction data. It is known that the bank number and terminal 


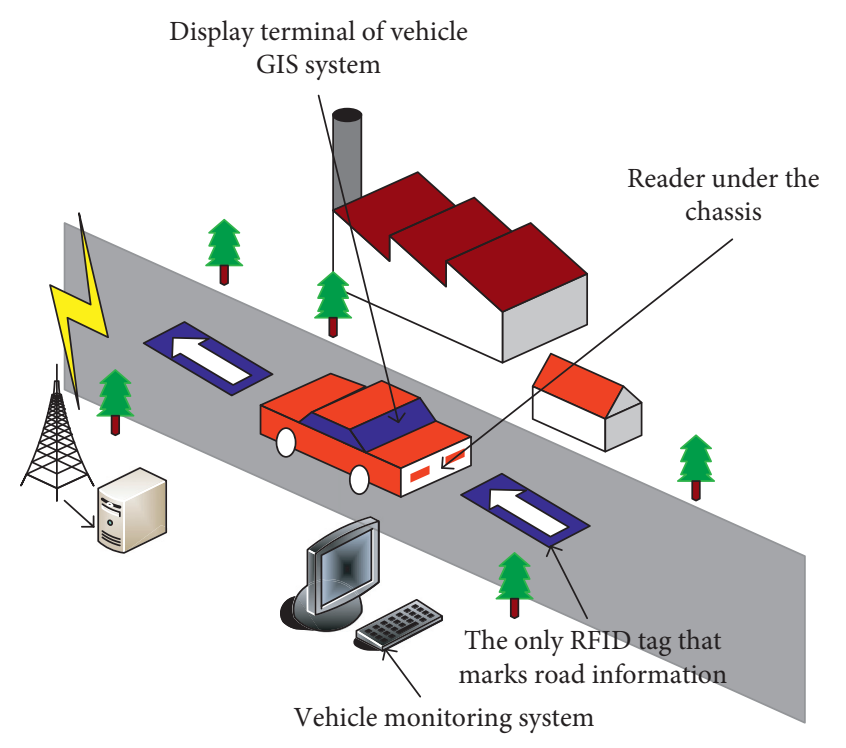

FIGURE 2: Taylor positioning principle.

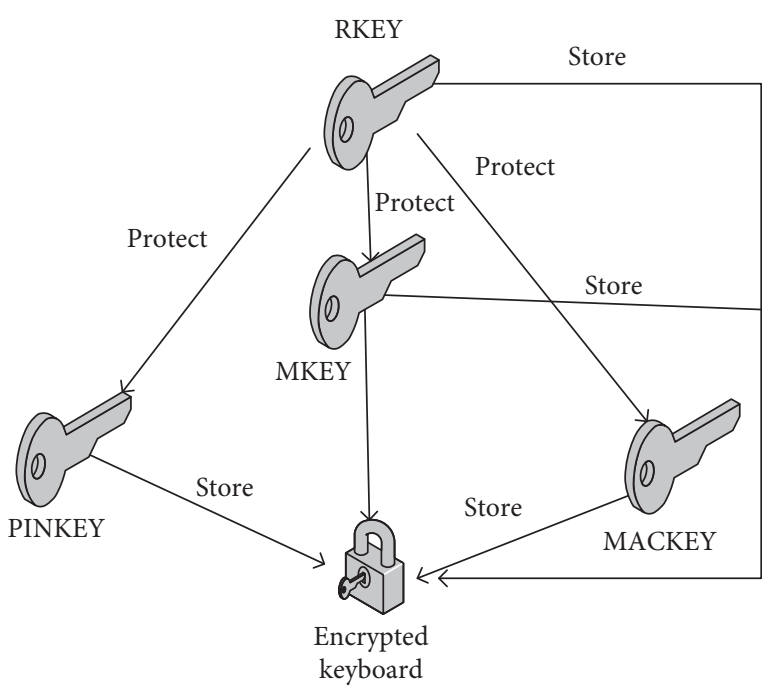

FIGURE 3: Key relationship structure.

number are bound one by one in the back-end database. That is to say, every time a terminal is added, the corresponding binding relationship must be added to the database; otherwise, the newly added terminal will not be able to perform operations and transactions.

(2) Secondly, use the PSAM card id number as the key to perform 3DES encryption on the first encrypted data. The PSAM card id number and terminal number are also bound one by one in the background database.

(3) Finally, use the random number generated by the PSAM card itself as the key to perform the last 3DES encryption on the second encrypted data, as Figure 5.

The BEBS system based on high security designed by this model solves the current problems of slow operation efficiency, large size, difficult monitoring, and low security of self-service terminals and has good scalability and maintainability, and the encryption settings are controllable, to maintain data, improve financial prevention and security, and play a long-term and stable role in the control of financial risks [14].

2.3. AHP Analytic Hierarchy Process. Aiming at the financial risk assessment system, the AHP analytic hierarchy process is used to construct a comparison and judgment weight matrix. The fuzzy weighted subset on $\mathrm{S}$ is set to $\mathrm{V}$, that is, from $\mathrm{V}$ to $\mathrm{Vn}$, where $\mathrm{V}$ is the index factor set $\mathrm{S}$ corresponding to the zth factor $S_{Z}$ of the weight .

(1) Construct a hierarchical structure.

(2) Construct a judgment matrix and fill in the judgment value.

According to the risk factors at the standard level, compare and analyze the index level and its related risk factors, and divide the rating according to the importance of the risk [15]. Here $\mathrm{x}_{\mathrm{zi}}$ is the importance level of the $\mathrm{z}$-th risk factor compared to the $\mathrm{i}$-th risk factor, and the value is assigned on a scale of $8 / 8$ to $22 / 2$, as shown in Table 1 .

From this, the matrix can be inferred: $R=\left(r_{z i}\right)_{m \times m}$.

(3) Calculate the weight index

$W_{\mathrm{z}}=\frac{1}{m} \sum_{j=1 a_{z j}}^{m} \sum_{k=1}^{\mathrm{m}}$

( $a_{\mathrm{zj}}$ refers to the elements of the judgment matrix, $1 \leq \mathrm{z}, \mathrm{j} \leq \mathrm{m}$ ).

(4) Consistency inspection

$$
\mathrm{C} . \mathrm{R}=\frac{\mathrm{Ci}}{\mathrm{Ri}} .
$$

When C R. $<1$, the consistency obtained $R=\left(r_{z i}\right)_{m \times m}$ is acceptable, when C R.>0.1, the matrix is unqualified and needs to be adjusted until the consistency is reached, and after calculation, when the order of the matrix is 1 and 2, its RI value is 0 , and the RI value will gradually increase as the order of the matrix increases. When the order of the matrix is 5, its RI values are both 1.19 . When the order of the matrix is 9, its RI values are all 1.47. It can be seen that this analysis method is available.

\subsection{Based on the Financial Risk Analysis Experiment and Results of the Internet of Things}

2.4.1. Simulation Experiment of DDoS Detection Module under NACO and TOCA Algorithm. In order to verify the convergence, detection rate, and false detection rate of the $\mathrm{NACO}$ algorithm that gives trust, some data in the KDD CUP99 13\% data set is selected and brought into the algorithm, and MATLAB 7.0 is used to simulate it. The KDD 


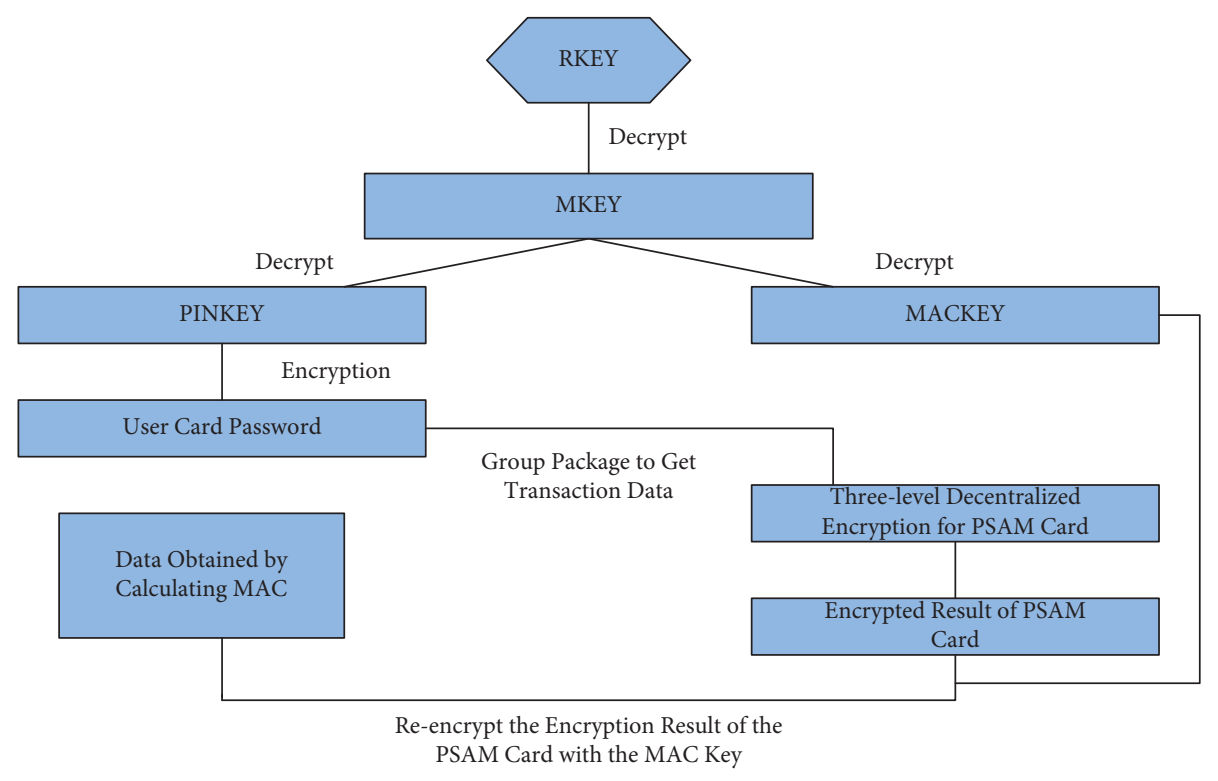

Figure 4: System encryption process.

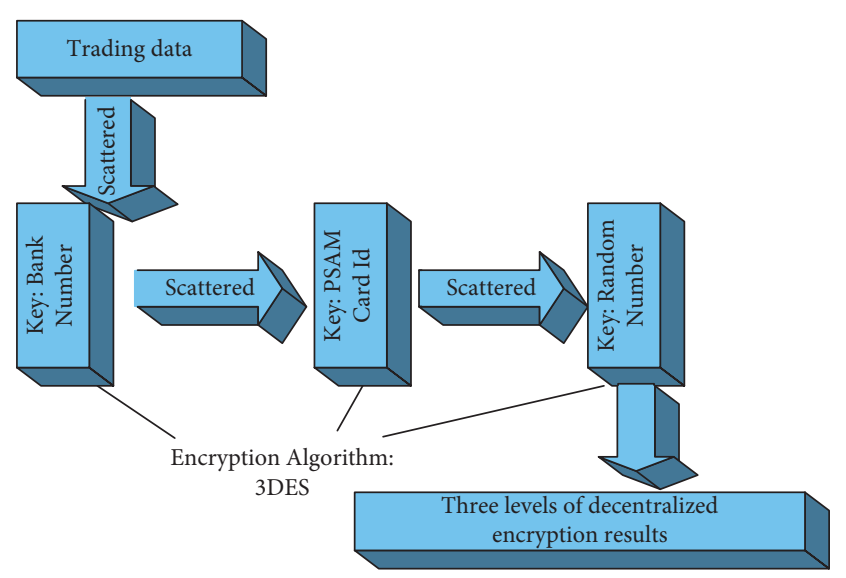

Figure 5: 3DES final encryption.

CUP99 data set is supported by the US National Defense Project and collected by MIT Lincoln Laboratory to collect real data. Randomly disperse $\mathrm{N}$ risk routing nodes in a square jurisdiction, and randomly select different proportions of risk nodes as malicious nodes. These malicious nodes can randomly launch black hole attacks and gray hole attacks in two forms of packet loss attacks [16]. KDD CUP99 13\% data set contains training data and test data, and both contain corresponding attack flags, which are suitable for testing the detection rate of detection algorithms. Then, in order to verify the pertinence of the model, the DoS attack stream data is selected for the abnormal data types that appear in the data set, including Neptune, smurf, udpstorm, and back [17]. In order to verify that the model has a learning function and has the ability to detect new types of attacks, the types of attacks in the training set are less than those in the test set. The number of training sets and test sets is 26375 and 13,563, respectively. 13\% of the data set contains many types of attacks, and each data set contains 39 parameter indicators, which can be divided into three categories: content characteristics, network characteristics, and traffic characteristics. In the network, the number of nodes is 18 to 99 (increments of 12 each time), and the proportion of malicious nodes is set to $13 \%$ (increases with the increase in the number of nodes); the number of nodes in the network is 66 , and the malicious nodes are set to $30 \%$. The proportion of nodes is 0 to $30 \%$ (increments of $6 \%$ each time). Through the simulation experiment when there is no malicious node, the average time required for its energy to return to normal is about $12 \mathrm{~s}$, provided that the energy of the risk node does not exceed the energy threshold, so the trust recovery time is set to $12 \mathrm{~s}$ in the simulation. Then two algorithms are used to train the nodes for detection rate and error rate.

After the training is completed, let us talk about the test set data input into the network. In order to ensure the accuracy of the experimental results, 12 repeated experiments were performed on the test set data, and the detection rate and false detection rate of the trust algorithm were compared with the detection rate and false detection rate of the TOCA algorithm, as Table 2 and Table 3.

Then through Figure 6 and Figure 7, it is clearly observed that the DDoS detection module is compared with the two algorithms through repeated experiments. The NACO algorithm shows a good detection rate and false detection rate. Then we can further measure the risk under the two algorithms and discuss risk factors.

\subsection{Risk Measurement Analysis under Different Algorithms of the Internet of Things}

2.5.1. Problem Description. Previously, we used various IoT solutions to calculate, detect, and monitor financial risks and explore risk factors. However, in order to further detect and calculate financial risk indexes, we will then combine multiple algorithms for comparison, verify through data, 
TABLE 1: Importance of risk factors.

\begin{tabular}{|c|c|c|}
\hline Number & Importance rating analysis & $\mathrm{x}_{\mathrm{zi}}$ assigned value \\
\hline 1 & Risk factors $z$ and $i$ are equally important & $1(8 / 8)$ \\
\hline 2 & The risk factor $z$ is slightly more important than $\mathrm{i}$ & $4 / 3(12 / 9)$ \\
\hline 3 & The risk factor $z$ is obviously more important than $\mathrm{i}$ & $8 / 3(16 / 6)$ \\
\hline 4 & The risk factor $z$ is much more important than $\mathrm{i}$ & $5(20 / 4)$ \\
\hline 5 & Risk factor $z$ is extremely more important than $\mathrm{i}$ & $11(22 / 2)$ \\
\hline 6 & The risk factor $z$ is slightly less important than $\mathrm{i}$ & $3 / 4(9 / 12)$ \\
\hline 7 & The risk factor $z$ is obviously less important than $\mathrm{i}$ & $3 / 8(6 / 16)$ \\
\hline 8 & The risk factor $z$ is much less important than $\mathrm{i}$ & $1 / 5(4 / 20)$ \\
\hline 9 & The risk factor $z$ is extremely less important than $\mathrm{i}$ & $1 / 11(2 / 22)$ \\
\hline
\end{tabular}

TABle 2: Detection data under NACO algorithm.

\begin{tabular}{lccc}
\hline Frequency & False positive (\%) & False negative (\%) & Accuracy (\%) \\
\hline 1 & 0.3154 & 0.0091 & 99.69 \\
2 & 0.3956 & 0.0000 & 99.60 \\
3 & 0.0001 & 0.0000 & 99.99 \\
4 & 0.5436 & 0.0000 & 99.55 \\
5 & 0.4345 & 0.0000 & 99.56 \\
6 & 0.2374 & 0.0000 & 99.76 \\
7 & 0.2859 & 0.0000 & 99.68 \\
8 & 0.2859 & 0.0000 & 99.68 \\
9 & 0.2859 & 0.0000 & 99.68 \\
10 & 0.2859 & 0.0000 & 99.68 \\
11 & 0.2859 & 0.0000 & 99.68 \\
12 & 0.2859 & 0.0000 & 99.68 \\
\hline
\end{tabular}

and propose a more complete algorithm. The financial risk algorithm is applied to the financial security and risk of the Internet of Things.

The financial industry has always been in the era of wind. As the pioneer of various industries, various technologies of the Internet of Things can be applied to the financial industry to see the spring of finance [18]. This article adopts the method of collecting data from authoritative publications, online portals, and magazines within the Sanfa financial regulatory agency, the People's Bank of China, the National Audit Office, the China Banking Regulatory Commission, and other institutions to collect data [19]. It is based on Internet of Things technology, different risk algorithms, risk detection, etc., to achieve the measurement of financial risks, to summarize and analyze and ensure effective monitoring and measurement of financial risks. At the same time, the results of the simulation experiment of the improved ant colony algorithm NACO are compared with the results of the basic ant colony algorithm TACO. As the optimization continues, the improved ant colony algorithm compares the searched optimal paths and will speed up the convergence when it is around the optimal solution [20] (Figure 8).

This figure shows the number of risk iterations horizontally, and it vertically shows the aging time. In the initial stage of risk, the pheromone on the link is relatively scarce, so the initial aging jitter fluctuates greatly, but as the number of iterations increases, the two algorithms measure aging jitter relatively stable. And from the comparison of Figure 8, the time delay jitter of the improved ant colony algorithm is generally lower than the risk measurement jitter of the basic ant colony algorithm TACO. Based on the comparison of stable parameters, it can be seen that the improved ant colony algorithm NACO is better in measuring failure jitter. The basic ant colony algorithm is $23.46 \%$ lower.

Then we respectively imagine different risk iteration frequencies based on the measurement results of the two algorithms of the Internet of Things. We use a network scenario designed with 32 hosts and 8 switches and set up 16 to 88 risk iteration trigger streams for transmission scheduling. We conduct simulation experiments on the NACO algorithm and compare the simulation results with the results obtained using the basic ant colony algorithm TACO. The above three algorithms are run 50 times, respectively, and the results obtained are shown in Table 4. It can be clearly seen from the table that the performance of the improved ant colony algorithm NACO is better than the basic ant colony algorithm TACO. Then we show the effect of different network topologies (the number of network links varies between 18 and 97) in the experiment and observe Table 4 to see.

With the gradual increase in the number of network links, the value of the objective function has increased, but it can also be seen that the increasing trend of the NACO algorithm is significantly lower than that of the TACO algorithm. In general, the optimal solution of the NACO algorithm is better than the TACO algorithm. Therefore, when financial risk measurement is based on the Internet of Things, the NACO algorithm can be preferred [21]. Based on this foundation, in this article we will use TSN scheduling for test analysis.

2.6. TSN Scheduling Algorithm with Incremental Strategy. We represent the financial network as a directed graph $\mathrm{Q}(\mathrm{U}$, $\mathrm{V})$, where $U \equiv(S \cup G)$ is a collection of nodes, and $E$ and $G$ are the collections of switches and hosts, respectively, and $\mathrm{V}$ represents the collection of links.

$V \equiv\{(z, j) \mid z, j \in U \boldsymbol{Z}, j$ Link Via Network Link $\} \quad$ represents a set of tuples of network links and expresses the risk measurement time trigger flow as a tuplet $_{z} \equiv\left(s_{z}, d_{z}\right), s_{z}, d_{z} \in G, s_{z}$ and $d_{z}$ separate the source and destination of the time flow. The set TS is the set of scheduled time-triggered flows, and each $i \in T S, d_{i}$ is the bandwidth requirement of the $\mathrm{i}$-th flow.

And the allocated capacity $(z, j) \in V$ for each link is Czj. In addition, for the bandwidth requirements of each link and each flow, a variable $a_{z j}^{i}$ is constructed. The variable $a_{z j}^{i}$ needs to meet the constraint, $a_{z j}^{i}=1$ or 0 ; it means that 
TABle 3: Detection data under TACO algorithm.

\begin{tabular}{lccc}
\hline Frequency & False positive (\%) & False negative (\%) & Accuracy $(\%)$ \\
\hline 1 & 0.0000 & 4.3147 & 94.772 \\
2 & 0.0000 & 8.2653 & 91.497 \\
3 & 0.0000 & 6.8124 & 93.417 \\
4 & 0.0000 & 14.3751 & 84.261 \\
5 & 0.0000 & 11.9262 & 88.065 \\
6 & 0.0000 & 13.8470 & 86.102 \\
7 & 0.0000 & 11.3339 & 89.341 \\
8 & 0.0000 & 9.9175 & 88.807 \\
9 & 0.0000 & 15.7144 & 83.162 \\
10 & 0.0000 & 6.2355 & 93.891 \\
11 & 0.0000 & 15.1315 & 80.25 \\
12 & 0.0000 & 4.6852 & 94.35 \\
\hline
\end{tabular}
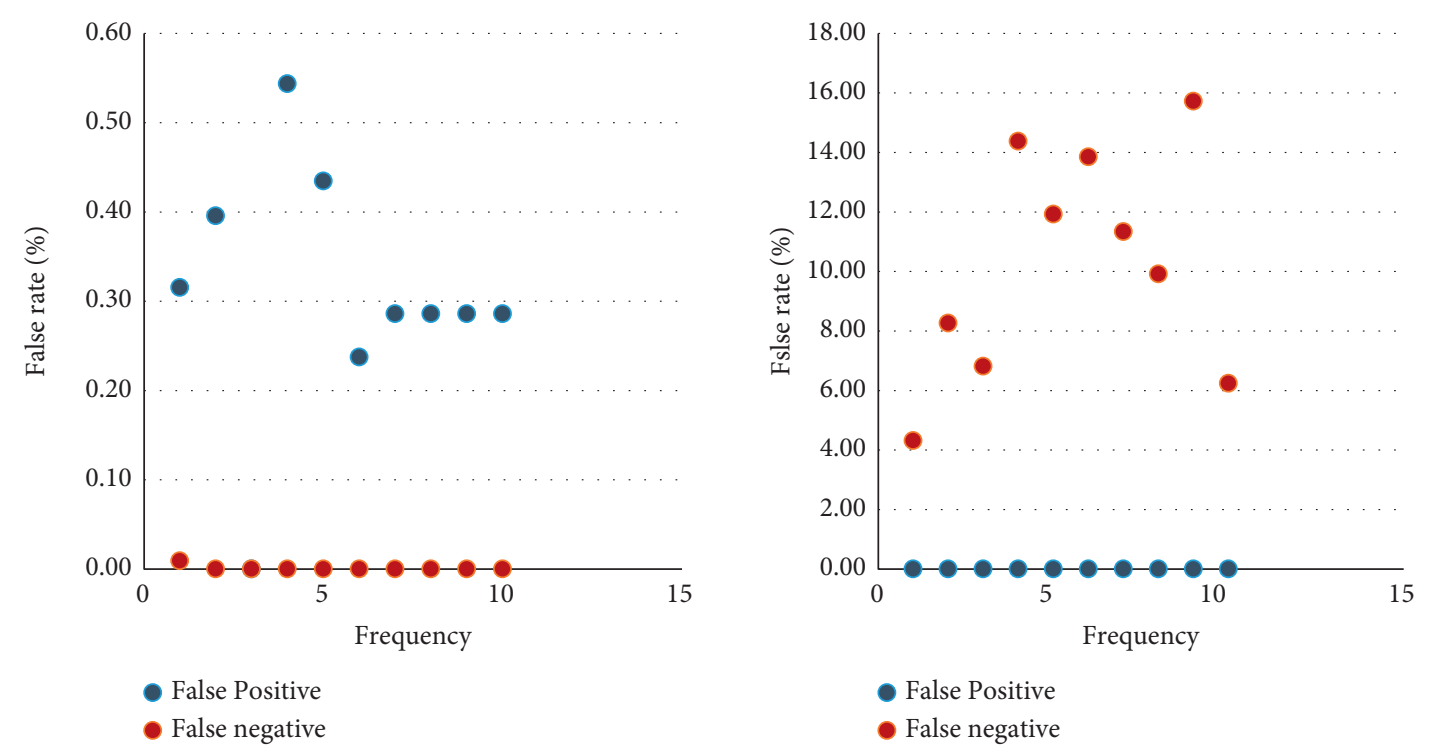

- False Positive

- False Positive

- False negative

Figure 6: Comparison of false detection rates of two algorithms.

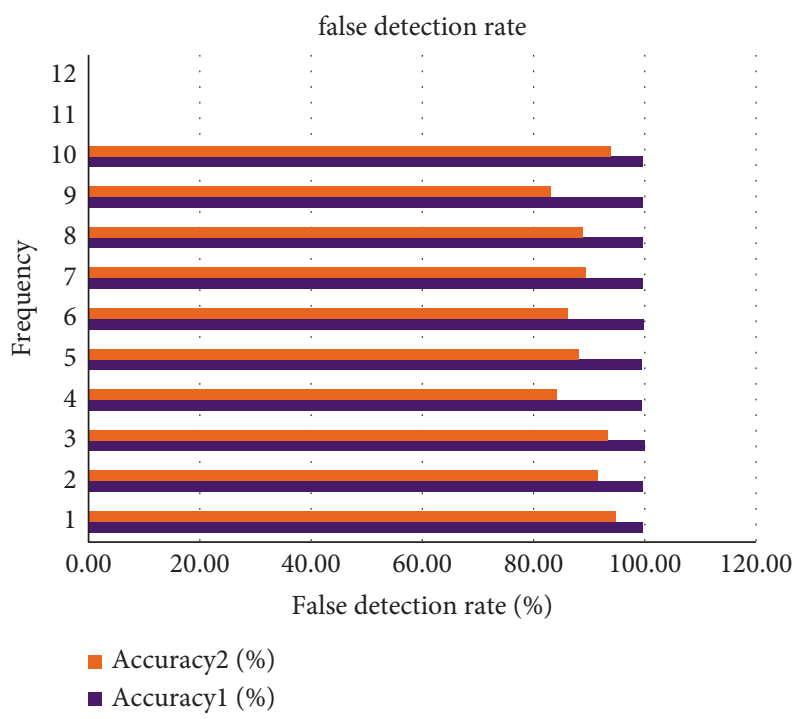

Figure 7: Comparison of the accuracy of the two algorithms. 

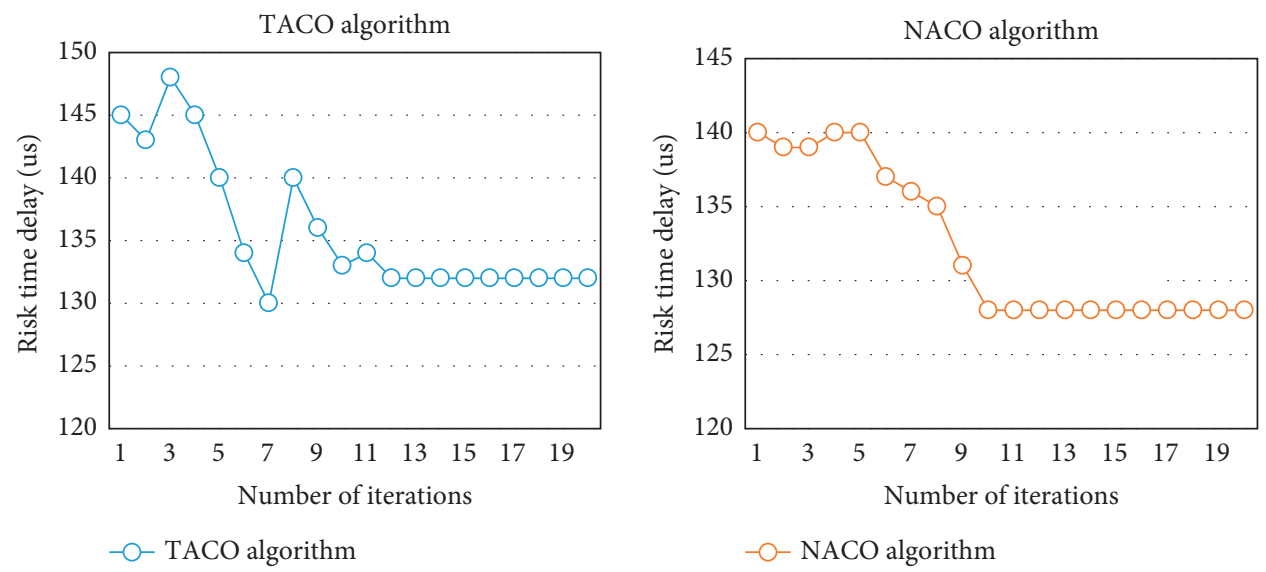

FIgURE 8: Two kinds of algorithm risk delay.

TABle 4: Metric under the two algorithms.

\begin{tabular}{|c|c|c|c|c|}
\hline Algorithm & Number of streams & Optimal value & Average value & Number of iterations \\
\hline TACO & \multirow{2}{*}{16} & 129.6 & 136.3 & 21.63 \\
\hline NACO & & 121.4 & 125.6 & 16.75 \\
\hline TACO & \multirow{2}{*}{29} & 134.3 & 138.4 & 23.11 \\
\hline NACO & & 123.7 & 127.7 & 16.98 \\
\hline TACO & \multirow{2}{*}{33} & 136.6 & 140.3 & 20.5 \\
\hline NACO & & 126.5 & 124.2 & 15.78 \\
\hline TACO & \multirow{2}{*}{47} & 139.4 & 142.2 & 18.79 \\
\hline NACO & & 129.5 & 131.1 & 15.33 \\
\hline TACO & \multirow{2}{*}{56} & 139.6 & 142.1 & 18.24 \\
\hline NACO & & 128.8 & 130.8 & 15.12 \\
\hline TACO & \multirow[b]{2}{*}{67} & 139.8 & 141.6 & 17.86 \\
\hline NACO & & 128.2 & 130.5 & 14.85 \\
\hline TACO & \multirow{2}{*}{75} & 140.1 & 141.7 & 17.11 \\
\hline NACO & & 127.9 & 130.2 & 14.52 \\
\hline
\end{tabular}

the required $i$ bandwidth provided by the link meets the demand, and the flow passes through the link, and the result is 1 , otherwise 0 .

Verify that the utilization of each link is lower than 1 and construct a check function.

$$
\sum_{i \in T S} \frac{d_{i} a_{z j}^{i}}{c z, j} \leq(z, j) \in V .
$$

Assuming that this calculation fails, this assumption must not be feasible because the final capacity limit of the link has been exceeded. However, if the test passes, there are two possibilities, schedulable and unschedulable [22] (continue to add until the program is not available), which is based on the incremental ant colony algorithm (I-NACO algorithm).

By showing the NACO algorithm and the I-NACO algorithm, the delay jitter changes the financial risk network in two scale scenarios. It can be observed from Figure 9 that the delay jitter fluctuations of the two algorithms are relatively large at the beginning. This is because the pheromone on the link is relatively scarce when the ants start to find the path. However, as the number of iterations increases in the two algorithms, the delay jitter of this algorithm tends to be relatively stable, has a definite delay jitter change, and can provide deterministic real-time guarantees for time-sensitive networks [23]. In scenario 2, the delay jitter of the I-NACO algorithm stabilizes faster than the NACO algorithm. The comparison of the two curves in Figure 10 shows that the delay jitter of the I-NACO algorithm is generally lower than that of the NACO algorithm. Based on the comparison of stable parameters, it can be seen that the delay jitter of the I-NACO algorithm is $7.1 \%$ lower than that of the NACO algorithm.

We set up different topologies (the number of network links varies between 5 and 55) and set up 75 risk measurement time-triggered streams for transmission scheduling and then measure the operation of different sizes of network topologies. It can be seen that with the linear increase of the topology size, the overall running time of the two algorithms is gradually increasing. For a smaller-scale network, the running time of the I-NACO algorithm is longer to a certain extent than the running time of the NACO algorithm, but for a slightly larger network topology, the running time of the I-NACO algorithm is shorter than that of the NACO algorithm, which shows that the I-NACO algorithm can measure financial risks in large-scale situations [24]. 

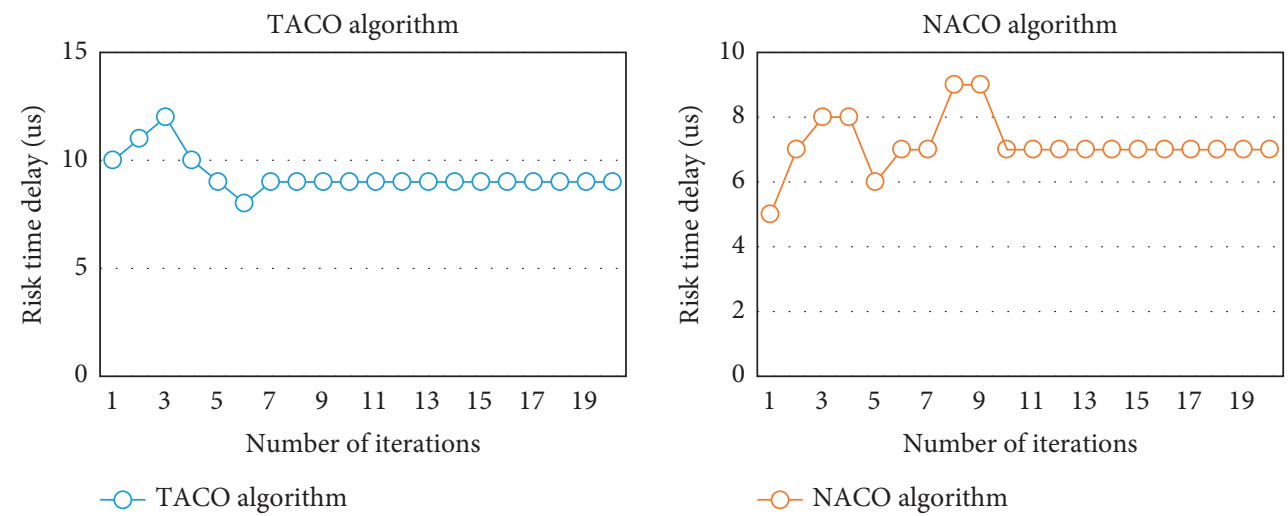

Figure 9: Two algorithms to measure timeliness.
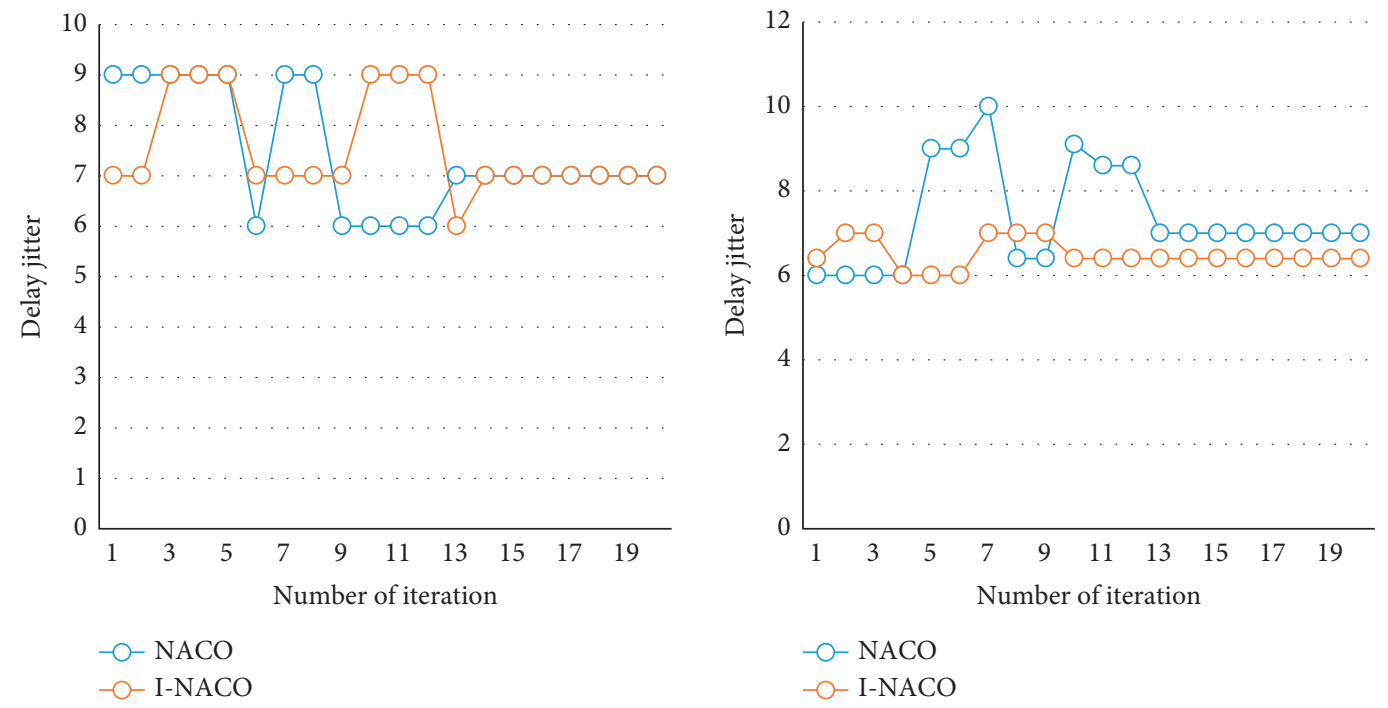

FIgURE 10: Delay jitter in different scenarios.

It can be seen from Figure 11 that this algorithm has significant utility for small-scale risk measurement. For large-scale risk measurement, there is no doubt that the incremental-based I-NACO algorithm shows more perfect utility. It can be seen that the TSN scheduling algorithm with incremental strategy can indeed occupy a place in large-scale risk measurement, and it can ensure the convergence speed and stability of the algorithm to the greatest extent [25].

\section{Discussion}

This article analyzes the Internet of Things environment, financial security, and risk exploration and calculations. First, it produces relevant backgrounds on the role of the Internet of Things and the role of the Internet of Things in finance. It explains three financial security events and conditions and discusses the risk section malicious node location methods and models and uses analytic hierarchy process to analyze risk factors, judge the risk level, verify different nodes as malicious nodes in simulation experiments, to convey to NACO and TACO intelligent degree of knowledge, detection rate, and misdetection rate, select DoS

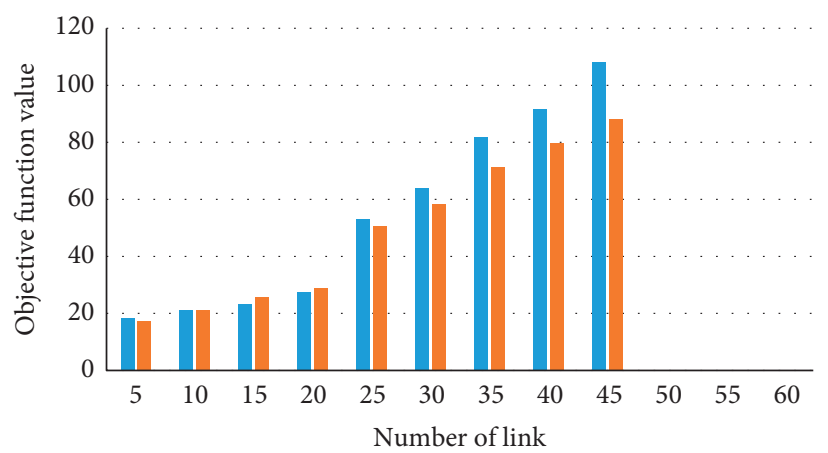

- NACO

- I-NACO

FIgURE 11: Metric under the two algorithms.

attack flow for the abnormal data type in the data set, simulate training, multiple experiments to get the data, 8 follow-up network scenarios designed with 32 hosts, and one switch, and set 16 goals coming soon to 88 . The 88 numbers to be formed will be used for transmission scheduling, calculate the NACO algorithm speed, and use the increment 
to obtain the TSN scheduling algorithm. Comparing the data, it can be seen that the I-NACO algorithm is against the NACO algorithm in potential capture, and the maximum speed and stability of the algorithm are guaranteed to a great extent. Also due to time and conditions, it is impossible to repeat more times and do more accurate experiments, but it can also bring a certain analysis effect based on the Internet of Things environment and financial risk research.

\section{Conclusions}

In this paper, we detect the financial risk changes in the IoT environment, measure the financial risk by various algorithms, and locate the malicious nodes in the risk segment, analyze the factors of risk occurrence, and judge the level of risk. And the recognition degree of the algorithm and the false detection rate are predicted on NACO and TACO algorithms. Through simulation experiment analysis, it is concluded that the NACO algorithm has a lower false detection rate and a better detection rate than the TACO algorithm for the measurement of financial risk under certain conditions. It is better than the basic ant colony algorithm in measuring failure jitter, $23.46 \%$ lower, and the optimized incremental I-NACO algorithm is $7.1 \%$ lower than the NACO algorithm in terms of delay jitter. The NACO algorithm has a significant effect on small-scale risk calculation, and there is no doubt that the I-NACO algorithm is more practical in large-scale risk measurement and can ensure the convergence speed and stability of the algorithm. It can not only save time to a certain extent, but also increase the accuracy of risk measurement without complicating the algorithm. In subsequent measurements, this optimization algorithm can be used for calculation.

\section{Data Availability}

No data were used to support this study.

\section{Conflicts of Interest}

The author declares that there are no conflicts of interest regarding the publication of this article.

\section{Acknowledgments}

The research was not funded.

\section{References}

[1] M. R. Palattella, M. Dohler, A. Grieco et al., "Internet of things in the 5G era: enablers, architecture, and business models," IEEE Journal on Selected Areas in Communications, vol. 34, no. 3, pp. 510-527, 2016.

[2] C. Perera, C. H. Liu, and S. Jayawardena, "The emerging internet of things marketplace from an industrial perspective: a survey," IEEE Transactions on Emerging Topics in Computing, vol. 3, no. 4, pp. 585-598, 2017.

[3] -sik Hyun and K-H Yoon, "FAIR-based BIA for ransomware attacks in financial industry," Journal of the Korea Institute of Information Security \& Cryptology, vol. 27, no. 4, pp. 873-883, 2017.
[4] Y. Fu, W. He, and R. Hao, "Comparative analysis of financial industry competitiveness of regions in China," Journal of Scientific \& Industrial Research, vol. 78, no. 1, pp. 11-14, 2019.

[5] K. Gai, M. Qiu, and H. Hassan, "Secure cyber incident analytics framework using Monte Carlo simulations for financial cybersecurity insurance in cloud computing[J]," Journal of Software Maintenance and Evolution Research and Practice, vol. 29, no. 7, pp. 1-13, 2017.

[6] R. Sato, Y. Hatanaka, Y. Ando et al., "High-speed operation of random-access-memory-embedded microprocessor with minimal instruction set architecture based on rapid singleflux-quantum logic," IEEE Transactions on Applied Superconductivity, vol. 27, no. 4, pp. 1-5, 2017.

[7] M. Filipe and Lins, "Register file criticality and compiler optimization effects on embedded microprocessor reliability," IEEE Transactions on Nuclear Science, vol. 64, no. 8, pp. 2179-2187, 2017.

[8] R. Tom and Halfhill, "Epyc embedded 3000 family adds ethernet, south bridge," Microprocessor Report, vol. 32, no. 2, pp. 19-24, 2018.

[9] C. Arnold, D. Kiel, and K. I. Voigt, "How the industrial internet of things changes business models in different manufacturing industries," International Journal of Innovation Management, vol. 20, no. 08, pp. 5-35, 2016.

[10] H. Mostafa, T. Kerstin, and S. Regina, "Wearable devices in medical internet of things: scientific research and commercially available devices," Healthcare Informatics Research, vol. 23, no. 1, pp. 4-15, 2017.

[11] M. A. Razzaque, M. Milojevic-Jevric, A. Palade et al., "Middleware for internet of things: a survey," IEEE Internet of Things Journal, vol. 3, no. 1, pp. 70-95, 2017.

[12] A. Xl and C. Dzb, "A meta-analysis on phenotypic variation in cadmium accumulation of rice and wheat: implications for food cadmium risk control," Pedosphere, vol. 29, no. 5, pp. 545-553, 2019.

[13] K. Gai, M. Qiu, and H. Hassan, "Secure cyber incident analytics framework using Monte Carlo simulations for financial cybersecurity insurance in cloud computing," Concurrency and Computation, vol. 29, no. 7, pp. e3856.1-e3856.13, 2017.

[14] J. Fan and A. Ruszczyński, "Risk measurement and risk-averse control of partially observable discrete-time Markov systems," Mathematical Methods of Operational Research, vol. 88, no. 4, pp. 1-24, 2018.

[15] R. Wang, C. Yu, and J. Wang, "Construction of supply chain financial risk management mode based on internet of things," IEEE Access, vol. 7, no. 99, pp. 110323-110332, 2019.

[16] R. P. Barneva, K. Kanev, B. Kapralos, M. Jenkin, and B. Brimkov, "Integrating technology-enhanced collaborative surfaces and gamification for the next generation classroom," Journal of Educational Technology Systems, vol. 45, no. 3, pp. 309-325, 2017.

[17] C. Farnsworth, L. T. Clark, A. R. Gogulamudi, V. Vashishtha, and A. Gujja, "A soft-error mitigated microprocessor with software controlled error reporting and recovery," IEEE Transactions on Nuclear Science, vol. 63, no. 4, pp. 2241-2249, 2016.

[18] S. Perandini, G. A. Soardi, M. Motton, A. Rossi, M. Signorini, and S. Montemezzi, "Solid pulmonary nodule risk assessment and decision analysis: comparison of four prediction models in 285 cases," European Radiology, vol. 26, no. 9, pp. 3071-3076, 2016.

[19] D. Carter, A. Charlett, S. Conti et al., "A risk assessment of antibiotic pan-drug-resistance in the UK: bayesian analysis of an expert elicitation study," Antibiotics, vol. 6, no. 1, p. 9, 2017. 
[20] H. M. Kim and M. Laskowski, "Toward an ontology-driven blockchain design for supply-chain provenance," Intelligent Systems in Accounting, Finance and Management, vol. 25, no. 1, pp. 18-27, 2018.

[21] J.-P. Briot, "From artificial neural networks to deep learning for music generation: history, concepts and trends," Neural Computing \& Applications, vol. 33, no. 1, pp. 39-65, 2021.

[22] E. Park, K. J. Kim, and S. J. Kwon, "Understanding the emergence of wearable devices as next-generation tools for health communication," Information Technology \& People, vol. 29 , no. 4, pp. 527-541, 2016.

[23] S. Nesnawy, L. M Gamal, A. Arafa et al., "Behavior-based exposure to droplet infection: development and validation of a self-risk measurement tool during the COVID-19 pandemic," Egyptian Journal of Health Care, vol. 11, no. 2, pp. 690-701, 2020.

[24] T. Kurosaki and Y. S. Kim, "Systematic risk measurement in the global banking stock market with time series analysis and CoVaR," Investment Management and Financial Innovations, vol. 10, no. 1, pp. 184-196, 2017.

[25] D. J. Armaghani, A. Mahdiyar, M. Hasanipanah, R. S. Faradonbeh, M. Khandelwal, and H. B. Amnieh, "Risk assessment and prediction of flyrock distance by combined multiple regression analysis and Monte Carlo simulation of quarry blasting," Rock Mechanics and Rock Engineering, vol. 49, no. 9, pp. 3631-3641, 2016. 